\title{
SOIL SAMPLING AUTOMATION CASE-STUDY USING UNMANNED GROUND VEHICLE
}

\author{
Eero Vaeljaots ${ }^{1}$, Henri Lehiste ${ }^{2}$, Meelik Kiik ${ }^{3}$, Tonu Leemet ${ }^{2}$ \\ ${ }^{1}$ Tallinn University of Technology, Estonia; ${ }^{2}$ Estonian University of Life Sciences, Estonia; \\ ${ }^{3}$ University of Tartu, Estonia \\ eero.valjaots@gmail.com, henri.lehiste@gmail.com,kiik.meelik@gmail.com, tonu.leemet@emu.ee
}

\begin{abstract}
The paper presents a case-study of a developed robotic soil sample collection system from bottom to top. While the sampling process automation is solved by using the robotic platform and electro-hydraulic mechanism, the control system is connected with cloud-based software that enables to create and manage operation tasks. Any soil fertility management program includes collecting the soil samples. Properly managing the test points and conducting the sampling process have great effect on the results. To enable more frequent sample collection, achieve better efficiency and quality, there is a great need for soil sampling automation. In this paper the robotic platform developed in the Estonian University of Life Sciences is adapted and modified to collect and store soil samples from fields and measure the soil parameters simultaneously. The platform navigates and operates autonomously with dedicated software and remote server connection. The software purpose is to enable flexible functionality during autonomous soil sampling operation, view detailed telemetry and create work tasks. Using sensor fusion, the navigation software calculates the optimal driving trajectories and prevents collision with obstacles. Also, integration with the test area management system is discussed, which enables definition of the test area and assigning sample points.
\end{abstract}

Keywords: soil, sampling, robot, vehicle, autonomous.

\section{Introduction}

Every soil fertility management program relies on properly collected soil samples as the most important input [1]. To solve a variety of agricultural issues, more elaborate soil maps are required and more information on different properties spatial variability is demanded. These include site-specific management, variable rate fertilization, soil quality assessment, natural resource monitoring, soil erosion risk mapping etc. Even landscape may result in uneven nutrient loads within a field [2]. Therefore, distribution of soil sampling points on the test area has great effect on the result quality [3]. Often surface sampling (up to $150 \mathrm{~mm}$ ) is not enough for deep-rooted crops and often sub-surface $(300-750 \mathrm{~mm})$ fertility needs to be analysed [4]. In addition to sample core being extracted, simultaneously other properties should be measured as compaction and soil moisture.

Usefulness and accuracy of precision farming techniques are heavily dependent on the soil sampling approach. As there is an increasing demand to collect more soil samples, the traditional manual methods are too laborious to conduct intensively. Besides that, sampling errors are much greater than analytical errors - any error during extraction tends to have cumulative effects and distort the soil maps and results. Conventional soil sampling consists of driving through the fields with an allterrain-vehicle (ATV) and manually probing the soil and collecting the samples to the container, Fig. 1.

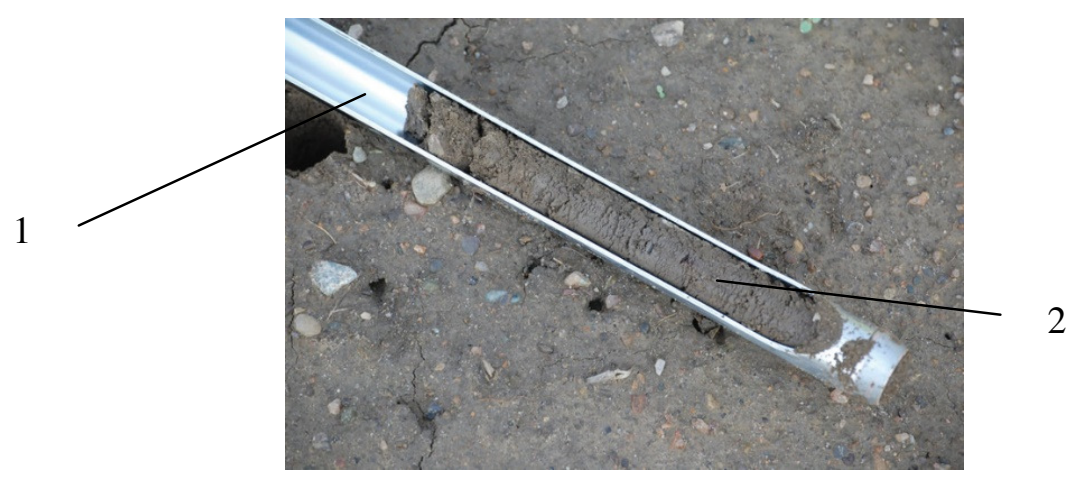

Fig. 1. Manual probe: 1 - sampling tube; 2 - extracted core

Already several semi-automated commercial solutions (Autoprobe, Magictec, Agriprobe, Falcon, Wintex) are in use, meaning the sample taking is automated, but the sampler is transported with an ordinary vehicle, while a human operator determines the trajectory. One of the most advanced, Autoprobe, in Fig. 2, is a device towed with a tractor through trajectories in the field and it 
automatically collects soil samples into the container using a probe mounted to the rubber track. Typically, they claim to double the probing speed in comparison with the human operator and manual process. Of course, in addition to hardware, dedicated software and management tools are needed for sampling pattern automation, which absence or low integration level might easily become a pitfall of the whole system.

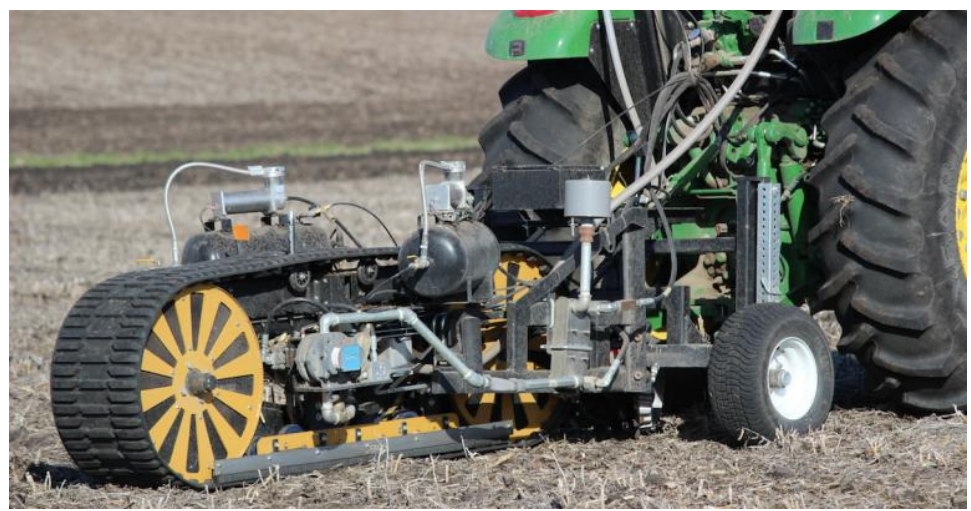

Fig. 2. Autoprobe automatic soil sampling device pulled by ordinary tractor

To achieve better efficiency and quality, there is a great need for soil sampling automation [5]. Being a monotonous and repetitive process for qualified personnel, it has suitable preconditions being conducted using an autonomously navigating unmanned ground vehicle (UGV). In this paper, the development of our automatic system is introduced and how cloud-based software system is implemented on mid-size UGV platform, which carries the soil sampling and storage apparatus. The novelty is the mechatronic system integration with control software. As a hypothesis, it is assumed that fully automated system under design is suitable to replace both traditional manual methods and semi-automatic systems.

\section{Materials and methods}

The articulated steering universal mobile robotic platform in Fig. 3 is developed in the Estonian University of Life Sciences in purpose of practical testing of unmanned technologies and navigation for agricultural activities [6]. The platform consists of multiple drive modules, interconnected by a central joint with 3 degrees of freedom. The current prototype is made up of 2 modules:

- first module serves as the platform for the mechanical drive system, which is powered by $15 \mathrm{~kW}$ Kohler internal combustion engine;

- second module is a platform for the soil sampling and storage mechanism and contains specialized hardware for autonomously performing sample probing, measurement of different soil properties, labelling and storing the sample.

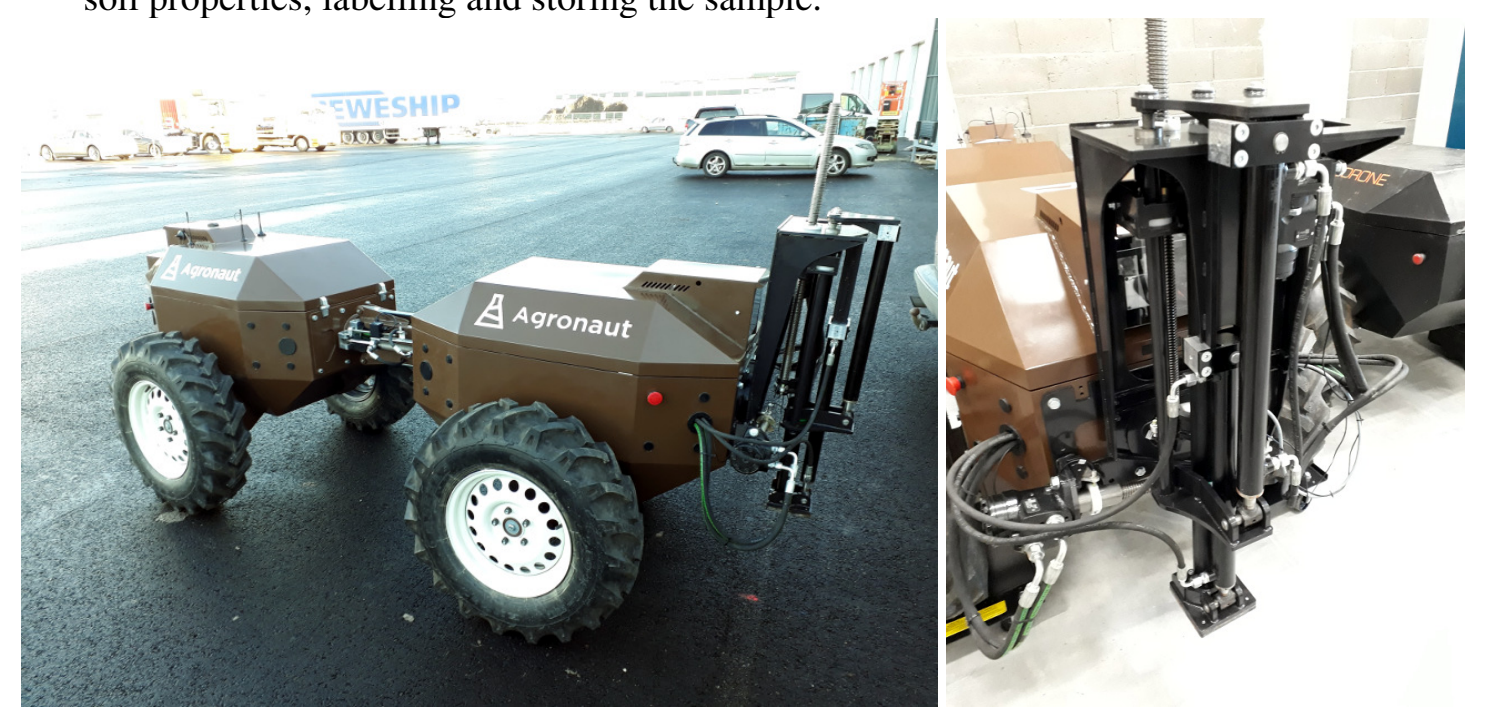

Fig. 3. Articulated steering UGV platform and soil sampling mechanism prototype 
UGV navigates with the aid of U-Blox Neo8 GPS unit and Bosch BNO055 IMU, each wheel and steering linkage encoders. Higher level obstacle detection uses "ZED” high-resolution stereo-cameras for range imaging complemented by "Leddar" inexpensive 3D lidars in front and back of the body for emergency system backup. Platform internal electronic modules in Fig. 4 use controller area network (CAN) for data exchange. The powerful Nvidia Jetson TX2 central processing unit (CPU) provides 20 $\mathrm{Hz}$ constant data output combined from data acquired from AVR based CAN modules. Data transmission with the server is possible over Wifi or $4 \mathrm{G}$ network.

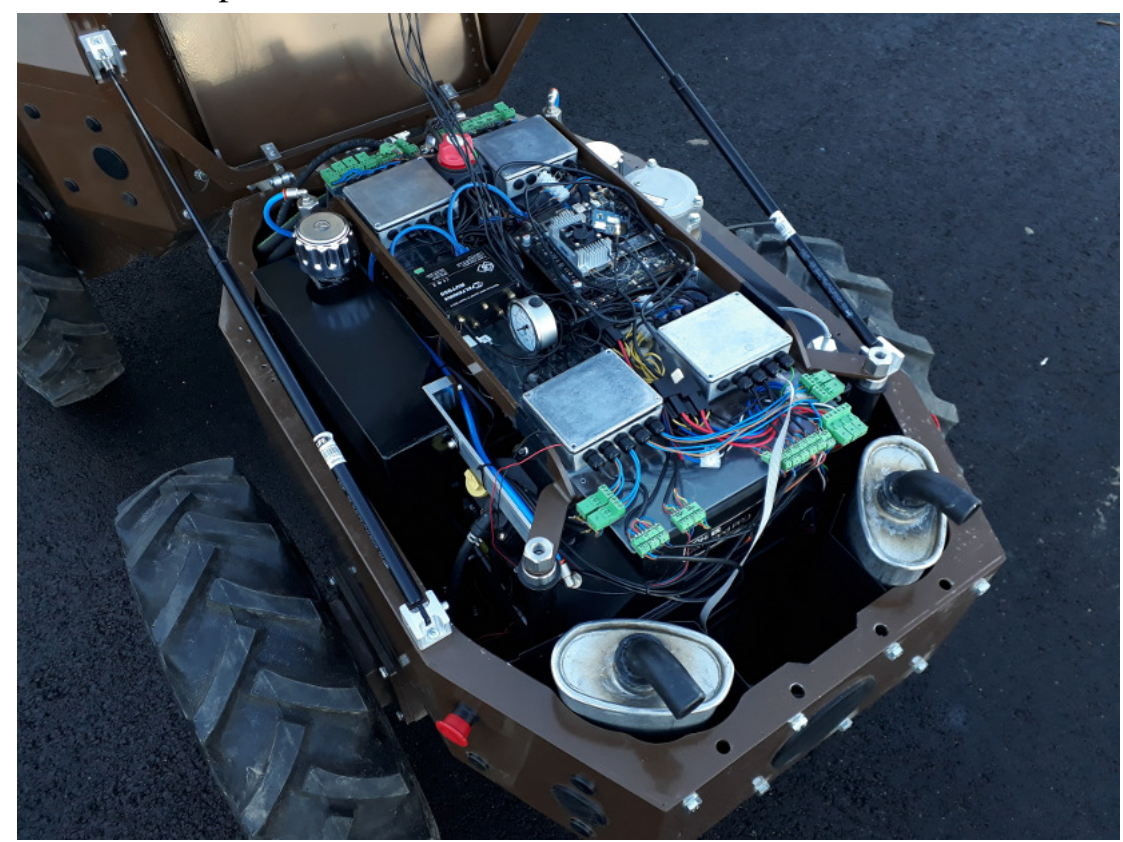

Fig. 4. Platform and soil sampling electronic system using CAN-modules

High speed systems that can be used without stopping the vehicle can take only short cores under $150 \mathrm{~mm}$ due to angular movement of the probe during operation. Here soil is needed to be probed deeper and other measurements are performed simultaneously (soil temperature and moisture measurement, penetrometer action) and the vehicle is required to stop, while the system is operated. Due to high force required for deeper core extraction, hydraulic actuators are used for the current solution. Soil sampling mechanism includes hydraulic actuators for lowering the probe to ground core extraction with constant speed, cleaning the probe and feeding the sample and manipulating the core. As only fraction of soil amount in core is taken to container due to volume restriction, it is important to use full core length, not only top or bottom. This is solved by adjusting the container end position under the probe, while the cleaner rod pushes collected core out of the probe. Screw mechanism is used for the probe to maintain constant process speed, therefore detect the resistance force from deceleration and calculate the penetrometer output using a 3-axis accelerometer.

For automating sample handling and storage, often the robotic manipulator arm is used [7]. The current solution uses simple stepper-powered 3 -axis coordinate system instead. The separate multiservo unit unpacks the standard carton container covers for sample feeding. The container block includes 140 slots for containers. For saving operation time, the storage system transports the carton containers only during the driving.

The calculation of sample points is done in Web applet "Agronaut", in Fig. 5, which includes Web UI for creating autonomous job layouts. Determining of the optimal order of sample points is solved by using shortest path algorithms, although usually manual alteration of points queue is often necessary. Usually the field is sampled using grid pattern covering points $0.2 \mathrm{ha}$ in a field of $2 \mathrm{ha}$ in size and sample taken from the middle of each area. The "Agronaut" outputs a shape-file in GeoJSON format, which is taken as an input in platform remote management software. This resulting shape-file contains definitions for job areas, job area transition paths and sampling waypoints in GPS coordinates. 


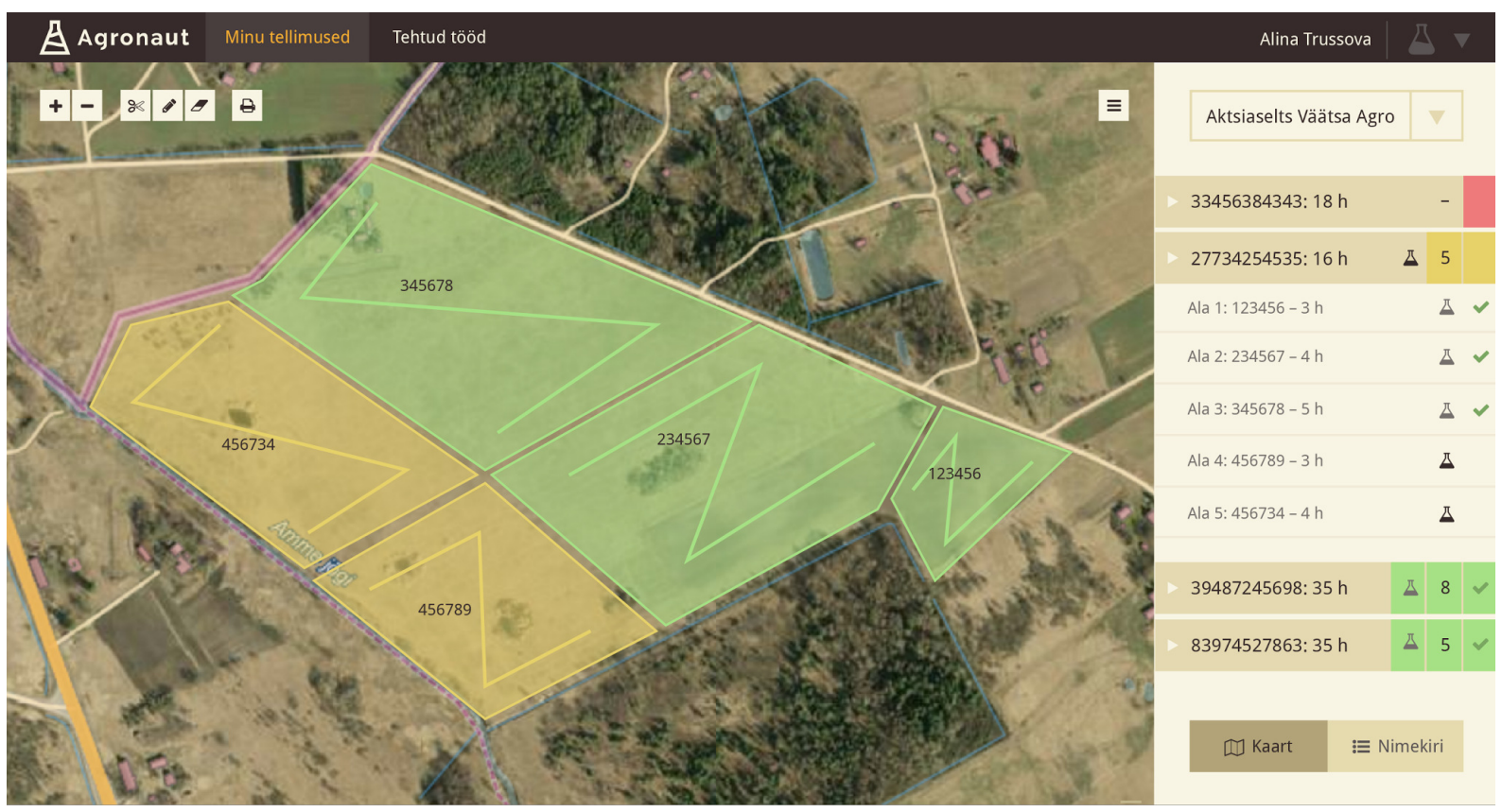

Fig. 5. Calculating optimal trajectories using applet

The platform remote management software purpose is to enable flexible functionality during autonomous soil sampling operations, analyse telemetry, create and manage robot work tasks, supervise the work process and enable manual control when needed, as shown in Fig. 6. It is based on NodeJS run-time environment and AngularJS framework. For achieving the maximum flexibility, the software uses service-oriented architecture. Platform firmware is divided into the following three parts, illustrated in Fig. 7.

1. Base functionality support module "libtuum" includes several standard libraries for utilization of Nvidia Jetson TX2 parallel cores;

2. Hardware control module implements controller area network (CAN) and hardware abstraction layer (HAL);

3. High level logic module includes application specific communication protocol and services.

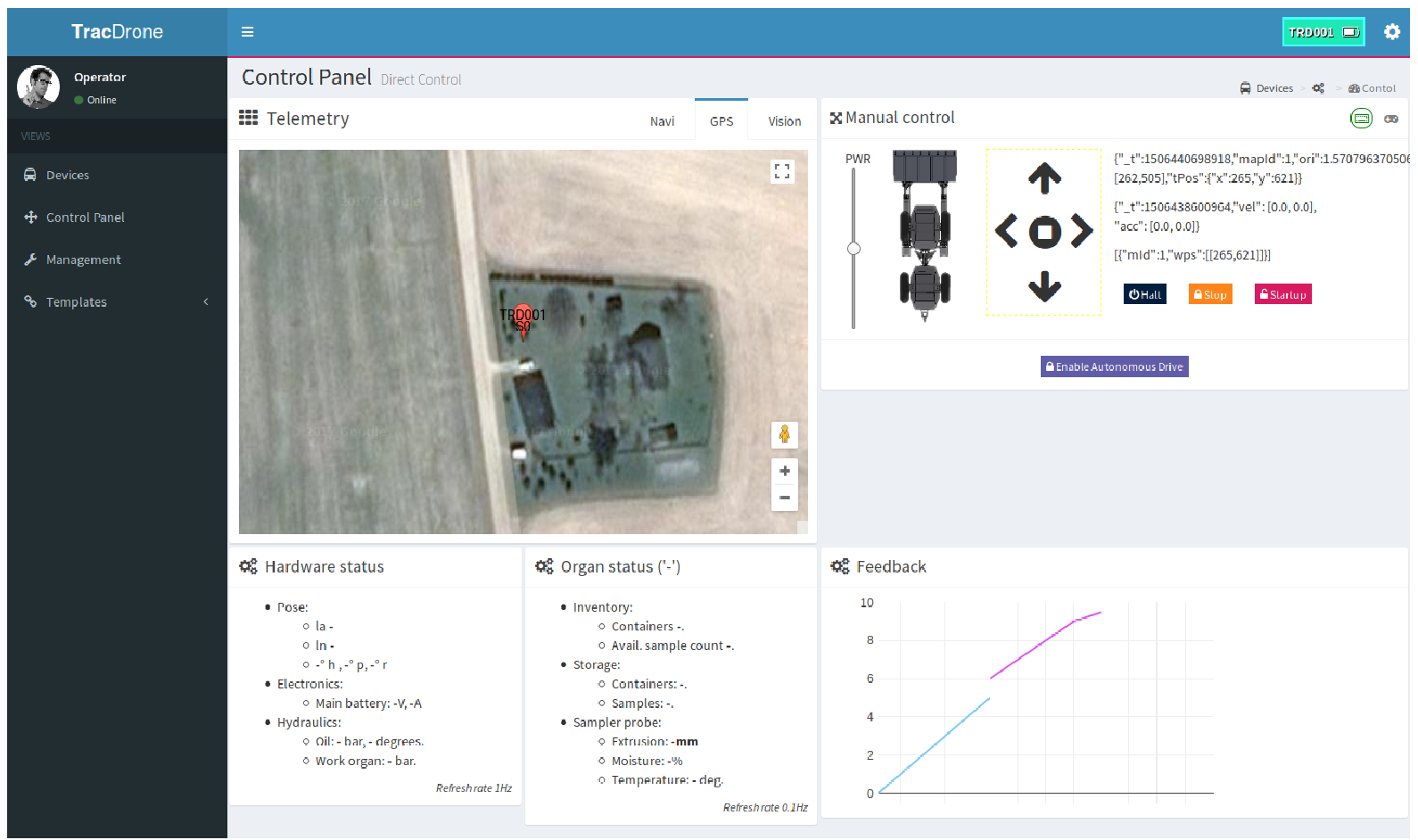

Fig. 6. Platform telemetry and control view 


\begin{tabular}{|c|c|}
\hline \multicolumn{2}{|c|}{ Base modules } \\
\hline tuum::Ipx & tuum::comm \\
\hline $\begin{array}{l}\text { Consolidated external library } \\
\text { interface module. }\end{array}$ & $\begin{array}{l}\text { WebSocket based server } \\
\text { containing a scalable } \\
\text { communication protocol } \\
\text { system. }\end{array}$ \\
\hline tuum::ogl & tuum::ocl \\
\hline $\begin{array}{l}\text { Shader language based } \\
\text { image processing pipeline } \\
\text { library based on OpenGL, } \\
\text { GLIP-Lib }\end{array}$ & $\begin{array}{l}\text { Data parallell processing } \\
\text { library base on the open core } \\
\text { language. }\end{array}$ \\
\hline tuum::hal & tuum::system \\
\hline $\begin{array}{l}\text { Hardware abstraction layer } \\
\text { interface. }\end{array}$ & $\begin{array}{l}\text { Subsystems runtime and } \\
\text { management service. }\end{array}$ \\
\hline tuum::vision & tuum::loc \\
\hline $\begin{array}{l}\text { Image pipeline interface } \\
\text { implementations and } \\
\text { processing algorithms library. }\end{array}$ & $\begin{array}{l}\text { Localization functionality } \\
\text { primitives module. }\end{array}$ \\
\hline
\end{tabular}

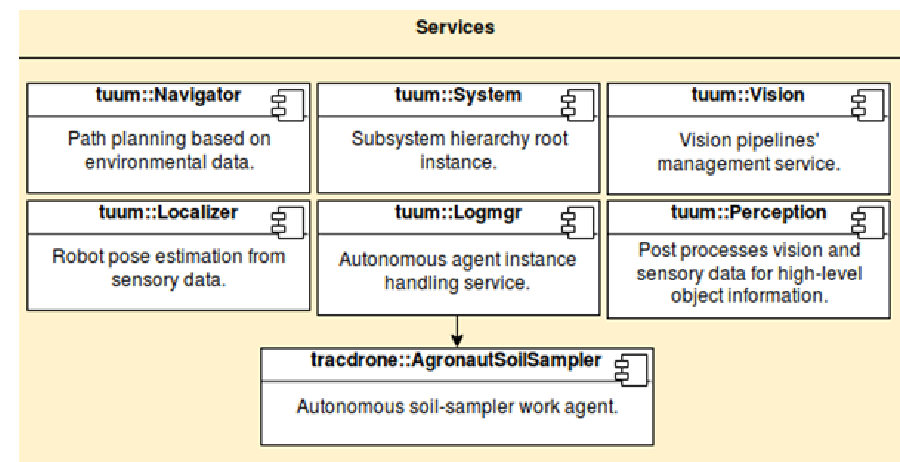

Fig. 7. Firmware modular layout

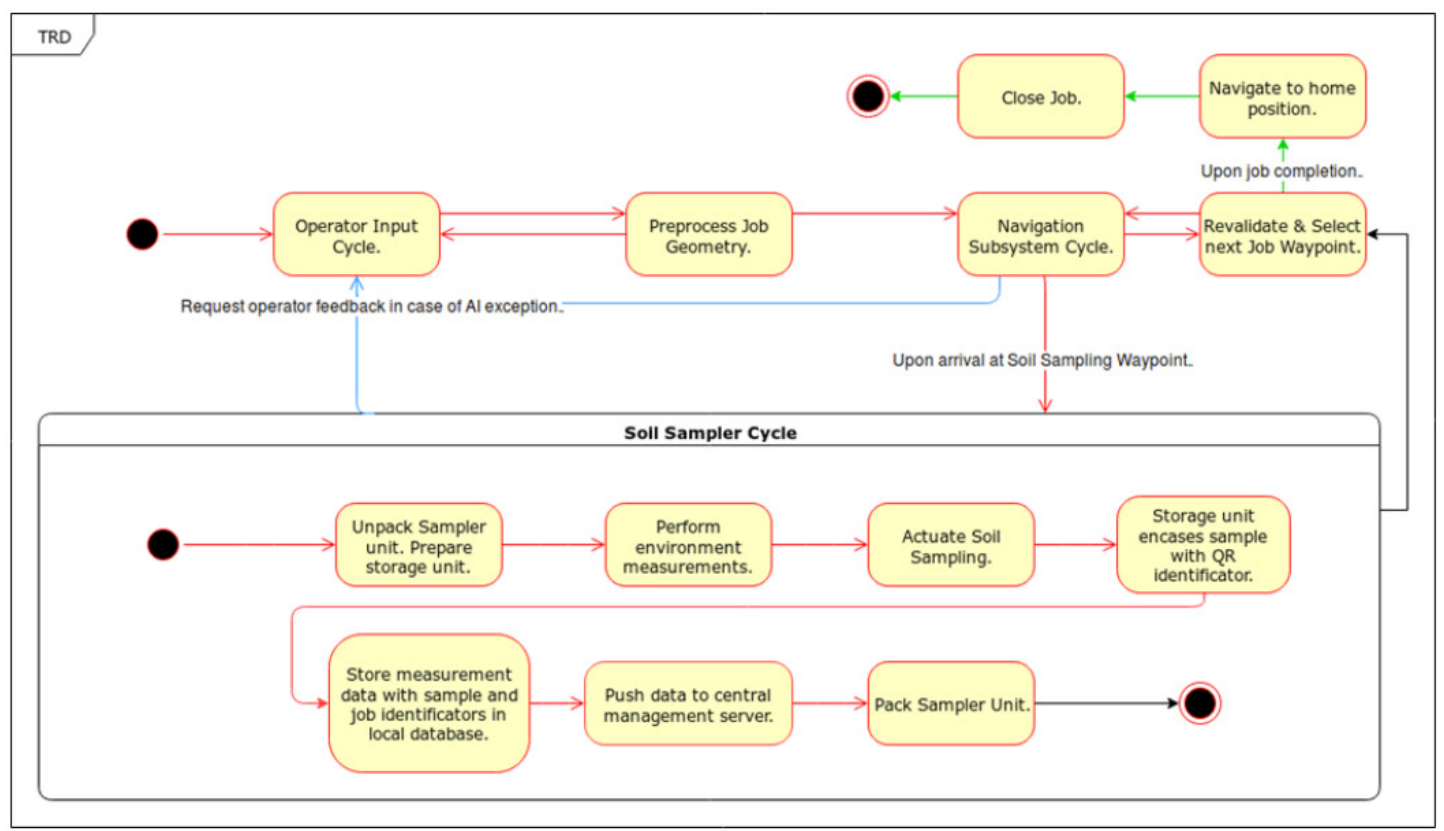

Fig. 8. Work cycle overview

UML diagram in Fig. 8 of the platform control unit work cycle explains the control unit firmware basic logic. While input shape-file is processed on the platform control system, coordinates are converted to Euclidean space, checks are done for out-of-bounds errors and defined navigation paths are checked against physical constraints of the platform. Furthermore, the defined job navigation paths are checked against local obstacle maps and alternative routes planned, if required. In the case of problems, feedback is requested from the operator in real time.

\section{Results and discussion}

The aim of the UGV project is to offer a fully automated platform that can be used to develop navigation algorithms for agricultural vehicles, as well as intelligent process planning algorithms and also multi-agent algorithms, when several platforms are used in co-operation. The measurement processes like soil sampling and soil parameter acquisition is one of the simplest processes to automate, as it does not involve object manipulation, like material handling and displacement etc.

Currently, the project is in proof of a concept state - it is verified that the composed system is working as expected. In comparison to the existing devices and methods that are used for soil sampling, the proposed system is attempting to automate every action during the process from the sample probing action to sample points locating and process planning. Therefore, besides supervision, the only action, where the operator is directly involved, is transporting the UGV to the field site. 
Combining several existing ready-to-use technologies and methods, a novel system is developed, demonstrating the integration of hardware and software components.

Operation speed as a performance indicator is measured with the sample taking time and driving time between the test points. Optimizing for speed is not the only concern, sampling quality is determined by how good representation of the soil composition the collected samples are. This is solved by test area management software, when the sampling path is generated.

Field tests are scheduled to spring, when the soil is dried up and the best soil sampling period starts. As the UGV navigation system is still experimental, navigation efficiency can be considerably improved as currently it is driving approximately 2-3 times slower than the operator with an ATV. The mean driving speed is $20 \mathrm{~km} \cdot \mathrm{h}^{-1}$ and the gasoline engine fuel consumption is $5 \mathrm{l} \cdot \mathrm{h}^{-1}$. However, the mechanical sampling system operating time is measured $6 \mathrm{~s}$ for obtaining one core, which is faster than the operator can do manually with the probe. Further testing includes comparison with a semiautomatic system fitted to a car trailer, provided by Agricon company.

Using automated software tools for test area management and for sampling operation setup combined with autonomous operation algorithms in the UGV control unit, takes much routine work away from the operator. Furthermore, additional instruments can be added to the sampler, enabling to acquire more information with less work. Currently the operator is required for verifying and modifying the proposed sampling schemas when needed, transporting the robot near the test area and process supervision.

\section{Conclusions}

1. A solution was proposed for automating the soil sampling process and mounted to mid-size mobile robot platform. Using cloud-based management and control software, the sampling process works automatically under the operator supervision.

2. Automating the traditional manual method has potential to speed up the process considerably. As the purpose of the current project is the concept and technical solution research, the system durability and efficiency can be improved further during the planned product development and testing.

3. Several soil parameters can be measured simultaneously with additional instruments: humidity, density, temperature etc. The results can be used to automatically update the field profile and maps in cloud application.

4. The project is in a proof of the concept state as the system is working. Field tests are scheduled to investigate the performance and efficiency of automated systems, as well as navigation algorithms.

\section{Acknowledgements}

This research was supported by funding of PRIA, project no. L160160TIPT "Autonoomse mullaproovide kogumise seadme väljatöötamine".

\section{References}

[1] Tan K.H. Soil Sampling, Preparation, and Analysis, 2nd ed. New York: CRC Press, 2005. 672 p.

[2] Logsdon S.D., Cole K.J. Soil nutrient variability and groundwater nitrate-N in agricultural fields. Science of The Total Environment, vol. 627, 2018, pp. 39-45.

[3] Biswas A., Zhang Y. Sampling Designs for Validating Digital Soil Maps: A Review. Pedosphere, vol. 28/1, 2018, pp. 1-15.

[4] Olson K.R., Al-Kaisi M.M. The importance of soil sampling depth for accurate account of soil organic carbon sequestration, storage, retention and loss. Catena, vol. 125, 2015, pp. 33-37.

[5] Krishna K.R. Push Button Agriculture: Robotics, Drones, Satellite-Guided Soil and Crop Management. Oakville: Apple Academic Press, 2016. 470 p.

[6] Väljaots E. Energy Efficiency Evaluation Method for Mobile Robot Platform Design. Tallinn University of Technology, PhD thesis, 2017. $96 \mathrm{p}$.

[7] Deusdado P., Pinto, E., Guedes, M. etc. An Aerial-Ground Robotic Team for Systematic Soil and Biota Sampling in Estuarine Mudflats. Robot 2015: Second Iberian Robotics Conference. Advances in Intelligent Systems and Computing, Springer, Cham, vol. 418, 2015, pp. 15-26. 